tères organoleptiques du lait réside dans l'abaíssement de la température, sont déjà une base d'une importance décisive pour des investigations ultérieures plus approfondies, qui seront entreprises au cours des prochains mois, avec des appareils de plus grande puissance et conçus de façon à obtenir le traitement du lait dans le liquide circulant. Le progrès très rapide réalisé dans la technique des ultra-sons au cours de ces deux dernières années permet de prévoir que le moment n'est pas loin où l'on pourra employer pour le lait et sur une grande échelle ce moyen physique énergiquement antimicrobien, qui possède entre autres le privilège d'agir à températures moyennes, sans causer aucune modification dans le " patrimoine" biologique du lait, et en grande partie dans sa constitution colloïdale, à part l'action bénéfique exercée sur la matière grasse.

\title{
TRAVAUX ET RECHERCHES SUR LE LAIT ET LES PRODUITS LAITIERS
}

\author{
(Fin.)
}

\section{MÉTHOdES ANALYTIQUES DU LAIT} (Section bactériologie) $(x)$

\section{Raisons de la création de la sous-commission de contrôle bactériologique du lait}

Alors que le contrôle des aérobies est depuis longtemps réalisé, on n'avait jamais pensé à organiser celui des anaérobies des laits crus, pasteurisés et des fromages. On s'est aperȩu des dangers de cette lacune à l'Armée d'Extrême-Orient, où une proportion très élevée de boîtes de fromage fondu ont été avariées par les anaérobies et en particulier par Pl. tetani.

Un comité de bactériologistes et d'industriels a été réuni pour réparer cette lacune.

Les travaux se sont déroulés parallèlement au Service des Anaérobies de l'Institut Pasteur et dans les laboratoires industriels.

\section{Recherches expérimentales et industrielles}

A. - Les faits cités plus haut d'une proportion très élevée de boîtes avariées de fromage fondu à l'Armée d'Orient montre que les anaérobies putréfiants et destructeurs peuvent s'y développer tardivement et entraîner la perte de denrées alimentaires. Il était logique de se demander en plus si le lait et ses dérivés pouvaient transmettre les maladies anaérobies.

(1) Rapport présenté par M. Prévot. 
Une série de recherches ont été faites pour répondre à cette question légitime et ont abouti aux conclusions suivantes :

1. Par voie parentérale, le lait peut transmettre au cobaye les six types de myosite gangréneuse : W. perfringens, $\mathrm{Cl}$. septicum, Cl. chauvooi, C. Oedemations, Cl. histolyticum et Cl. sordellii, les cinq types de botulisme A, B, C, D, E, et irrégulièrement le tétanos.

$2^{\circ}$ Par voie entérale, le lait peut transmettre au cobaye les six types de viscérites correspondant aux six myosites sus-nommées ; les cinq types de botulisme, mais jamais le tétanos.

$3^{\circ}$ Le contrôle bactériologique du lait doit donc comporter la détection des anaérobies.

B. Recherche d'une méthode pratique de détection des anaérobies. Les travaux faits à l'Institùt Pasteur sur la réduction des sulfites par les anaérobies ( 2 et 3 ) ont permis de transposer rapidement la technique des géloses sulfite + fer sur le plan pratique pour cette recherche. Au laboratoire, nous avons d'abord vu que cette technique permettait de réisoler de laits préalablement infectés tous les anaérobies étudiés dans la partie $\mathrm{A}$ de nos recherches.

Cette méthode enseignée aux techniciens des industries du lait a été très vite appliquée par eux et a donné d'excellents résultats. Elle a d'abord montré l'abondance des anaérobies dans les laits erus et leur persistance, quoique dans une proportion très diminuée, dans les laits pasteurisés. Ce premier fait rejoint les travaux canadiens récents de PAQUET et GAUvin [4] qui ont vu que la recherche des Clostridium gazogènes (par la méthode chauffage à $85^{\circ}$ ) détectait $6,4 \%$ d'anaérobies dans les laits crus, et $5,3 \%$ dans les laits pasteurisés parmi lesquels $28,2 \%$ de perfringens dans les premiers et $18,3 \%$ dans les seconds. Ils en concluent que les règlements concernant le contrôle bactériologique du lait devraient être modifiés.

C'est aussi à cette conclusion que nous aboutirons quand nos méthodes seront bien au point et auront fait leur preuve à l'échelle industrielle.

C. Etude bactéristique du lait et du fromage fondu. - Cinq envois de colonies noires nous ont été faits. Ce qui nous a permis d'établir un début de bactéristique du lait. Dans le tableau ci-après nous, donnons les résultats positifs (de nombreux échantillons ne contenaient que des anaérobies facultatifs qui n'ont pas été déterminés).

\section{Résumé}

Sur 22 envois ayant fourni 22 souches anaérobies strictes, on a trouvé :

Flore nécessaire : $W$. perfringens, 3 fois ; C. sporogenes, 3 fois ; 


\begin{tabular}{|c|c|c|c|}
\hline Provenance & Date & Désignation & Résultats \\
\hline M. Mocquot & Juin 1952 & Fromage fondu & Cl. sporogenes \\
\hline M. Mocquot & Juin 1952 & Fromage fondu & $\mathrm{Cl}$ sporogenes \\
\hline « & « & \& & « \\
\hline Vét. Cdt Houzé & Juillet 1952 & Fromage fondu Bel & Eubact. quartum. \\
\hline " & $x$ & a & * \\
\hline " & \& & « & $\alpha$ \\
\hline * & « & - & $x^{3}$ \\
\hline " & * & * & $-x^{3}$ \\
\hline " & * & v & a \\
\hline " & " & 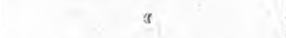 & 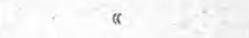 \\
\hline$\alpha$ & " & " & $\&=-$ \\
\hline * 4 & * & " & $\propto$ \\
\hline$=\pi$ & " & * & - \\
\hline " & « & « & * \\
\hline M. Pren & Août 1952 & Lait pasteurisé & W. perfringens \\
\hline$\pi$ & a & Présure & Cl. bifermentans \\
\hline « & Décembre 1952 & Fontenay & W. perfringens \\
\hline - $\quad$ " & " & Lait stérilisé & Cl. valerianicum \\
\hline * & $\bar{c}$ & Ablis & W. perfringens \\
\hline M. Mocevot & Décembre 1952 & Fromage fondu & Cl. carnofoetidum \\
\hline is & $"$ & « & Cl. sporogenes \\
\hline$=$ & " & " & Cl. valerianicum \\
\hline * $\quad *$ & " & a & Cl. valerianicum \\
\hline
\end{tabular}

Cl. valerianicum, 3 fois ; Cl. bifermentans, 1 fois ; Cl. carnofotidum, 1 fois.

Flore contingente: Eub. quartum, 11 fois.

Ainsi la flore des laits crus et pasteurisés, qui persiste dans le fromage fondu est exactement la flore originelle universelle (5 et 6 ) avec ses deux composantes : nécessaires (sporulée) contingente (asporulée). Ceci permet d'affirmer que les laits et les fromages sont souillés par la terre.

Ces recherches sont très longues; actuellement le travail de RAIBAUD [7] permet d'envisager qu'on pourra mettre au point dans un proche avenir des réactions biochimiques rapides qui permettront de dire que tel lait est souillé par cette flore originelle universelle : grâce aux réactions permettant de déceler rapidement la substance indolique trouvée dans les cultures d'une variété de $C l$. sporogenes du lait et du fromage fondu, on pourra imiter les méthodes qui permettent de dire si une eau est polluée par certains microbes.

Conclusions. - Une méthode efficace de détection des anaérobies 
stricts du lait et des fromages a été appliquée. Elle permet la numération globale des germes et la détermination des espèces en cause. Elle permettra de trouver des réactions biochimiques rapides pour déceler les espèces fréquentes et dangereuses.

\section{MÉTHODES ANALYTIQUES DU LAIT (Section chimie) (I)}

Les méthodes d'analyses des laits dont nous avions annoncé la publication imminente au cours de notre réunion du 17 décembre 1951 ont paru dans les Annales de la Nutrition et de l'Alimentation (1951, V, 537).

Soumises par l'A.F.N.O.R. à l'enquête publique, elles ont été adoptées pratiquement sans modification. D'autre part, elles ont servi de base aux travaux d'une Commission technique créée au sein du Service de la Répression des Fraudes et qui avait pour but d'élaborer de nouvelles méthodes officielles. Cette Commission a, pour une large part, adopté nos conclusions.

Ces premiers résultats acquis, une modification de nos méthodes de travail s'imposait qui nous permettrait d'approfondir les points dont nous avions négligé l'étude, faute de temps, M. le Professeur TerroIne nous a conseillé de choisir quelques sujets parmi ceux qui nous paraissaient les plus importants ou les plus urgents et de constituer de petits groupes de travail chargés d'accomplir les recherches nécessaires pour justifier le choix ultérieur de la Commission.

Les sujets mis à l'étude sont relatifs aux laits et aux laits concentrés ou en poudre. Voici quels sont ceux pour lesquels a été créé un groupe de travail et les premiers résultats obtenus :

$1^{\circ}$ Choix d'une méthode de référence pour le dosage de la matière grasse et d'une méthode de détermination des matières sèches (1). Responsable : M. NAveliter, Ingénieur au Laboratoire Municipal de Paris.

Pour le dosage de la matière grasse, nous avons adopté la méthode Röse-Gottlieb retenue comme méthode étalon par la Fédération Internationale de Laiterie. Mais, ainsi d'ailleurs qu'il a été précisé à la dernière réunion de cet organisme qui s'est tenue à Madrid en septembre dernier, cette méthode n'est sûre que si on l'applique au lait frais. Or, les experts et les services de contrôle ont souvent à analyser des laits altérés ou additionnés d'un antiseptique susceptible de gêner le dosage (e'est, en particulier, le cas du formol). Cela justifie l'étude d'autres méthodes : celle du Laboratoire Municipal de Paris a fait l'objet de nombreux essais. Ajoutons

(1) Rapport présenté par $M^{1} \mu_{e}$ Bejambes. 
qu'elle a été proposée comme méthode officielle par le Service de la Répression des Fraudes.

Pour la détermination des matières sèches, nous avons gardé provisoirement l'ancienne méthode officielle ( 7 heures de chauffage au bain-marie bouillant), mais nous en connaissons toutes les imperfections et d'autres méthodes (en particulier dessiccation en s'aidant du vide) sont actuellement à l'essai.

$2^{\circ}$ La question des laits altérés. Responsable : M. Navellier.

Les méthodes d'analyse que nous avons publiées sont valables pour les laits frais ou en bon état de conservation. C'est négliger une bonne partie des échantillons que les experts et les services de contrôle ont à examiner. On sait en effet dans quel état d'altération plus ou moins avancée se trouvent certains laits soumis à l'expertise.

Le groupe de travail chargé de cette question estimant que le plus simple serait évidemment d'éviter ou tout au moins de limiter cette altération, a rédigé un vœu demandant que soit apportée plus de rapidité dans les expertises. Ce vœu a été transmis, il y a environ un an, par les soins de M. le Professeur Terroine, au Ministère de la Justice. S'il se révèle inefficace, le problème restera : comment traiter un lait altéré ? Quelles conclusions tirer des résultats de l'analyse?

1ํㅛ Un premier travail très intéressant a été effectué : $\mathbf{M}^{\text {me }} \mathrm{RuIz}$, Ingénieur au Laboratoire Municipal de Paris, a étudié le dosage du lactose dans les échantillons vieillis de lait de vache. Mme RuIz a trouvé sur les laits additionnés de conservateurs et gardés pendant un temps plus ou moins long, après leur prélèvement, des doses de sucres réducteurs, exprimées en lactose, d'un lait de vache, le chiffre calculé d'après une détermination réductimétrique étant toujours supérieur à celui donné par polarimétrie.

Ces différences sont dues à la présence, dans les lacto-sérums des laits considérés, de glucose et de galactose résultant de l'hydrolyse partielle du lactose. $M^{m e}$ RuIz estime donc que pour déterminer arec certitude l'exacte teneur d'un lait en lactose, il est nécessaire d'en effectuer l'hydrolyse totale, puis de doser par polarimétrie ou réductimétrie glucose et galactose.

$3^{\circ}$ Antiseptiques et neutralisants (1). Responsable : M. le Commandant Rovquetre, Chef du Laboratoire de Chimie de l'Inspection technique des Subsistances de l'Armée.

Je cite seulement pour mémoire ce groupe de travail dont la création est trop récente pour qu'il ait pu encore aboutir à des résultats. 
$4^{\circ}$ Dosage du saccharose dans le lait concentré sucré (1). Responsable :

M. Vulllaume, Professeur à l'Ecole Nationale Vétérinaire d'Alfort.

Ce sujet a fait l'objet d'une publication de M. Vulluaume dans la revue Le Lait $(1952,32,600)$. On sait que ce dosage qui paraît simple a priori est en réalité compliqué par le fait que le saccharose ajouté peut avoir subi, avant ou après son addition au lait, une hydrolyse partielle non négligeable.

M. Vuillaume provoque l'hydrolyse complète des holosides, lactose excepté, puis une oxydation des glucides aldéhydiques par l'iode en milieu alcalin. Du taux de lévulose dosé par euprimétrie, il peut déduire le saccharose initial.

$5^{\circ}$ Dosage de la matière grasse dans les laits en poudre. Responsable : M. Pren, Directeur des Laboratoires de la Société des Fermiers réunis.

Pour le dosage de la matière grasse dans les laits en poudre, le groupe de travail avait à sa disposition un certain nombre de méthodes : méthode officielle anglaise, méthode officielle américaine, méthode Röse-Gottlieb classique, méthode de Röse-Gottlieb modifiée, méthode S.B.R.). Pour nous permettre de fixer notre choix, M. Pien nous a proposé de doser par ces différentse méthodes la matière grasse d'une poudre de lait dont il connaissait exactement le taux butyreux, car elle avait été préparée spécialement avec un lait dont la richesse exacte en matière grasse avait été déterminée. Plusieurs laboratoires, officiels et privés, ont effectué ces dosages. Tous ont trouvé que la méthode officielle anglaise donnait les valeurs les plus voisines des valeurs théoriques, tout en fournissant une matière grasse exempte d'impuretés.

Sur des poudres de lait de taux butyreux inconnu, elle a donné les chiffres les plus forts. Enfin, elle convient aussi bien aux poudres Hatmaker qu'aux poudres Spray et à celles qui ont été additionnées de sucre.

Le groupe de travail a done décidé d'adopter cette méthode modifiée seulement en ce qui concerne l'appareillage.

(La méthode officielle anglaise consiste en attaque successive de la poudre par de l'eau ammoniacale et de l'acide chlorhydrique, puis dissolution de la matière grasse par le mélange d'éther sulfurique et d'éther de pétrole.)

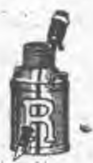




\title{
BIBLIOGRAPHIE
}

[1] Prévot et Thouvenot. Ann. Inst. Past., 1952, 83, 180.

[2] Prévot. Ann. Inst. Past., 1948, 75, 571.

[3] Prévot, Saissac et Callame. Ann. Inst. Past., 1950, 79, 93.

[4] Paquet et GaUvin. Canad. Journal Health., 1953, 44, 92.

[5 ]Prévot et Moureav. Ann. Inst. Past., 1952, 82, 13.

[6] PrÉvot. La Nature, 1952, no 3.205, p. 147.

[7] Ratbatd, Mémoire de Concours.

\section{SEMBLE-T-IL CONVENABLE DE MODIFIER LES MÉTHODES DE TRAVAIL DES DÉPOTS CENTRALISATEURS DE LAIT DE FEIMIME EXIGÉES ET UTILISÉES JUSQU'A PRÉSENT?}

\author{
par
}

\section{K. KAYSER}

L'opinion des pédiatres allemands que le lait de femme est la meilleure source de nourriture pour les nouveau-nés et les nourrissons fait toujours autorité, et les laits humanisés de vaches n'ont pas encore prouvé d'une façon convaincante leur supériorité.

Aussi longtemps qu'il en sera ainsi, les dépôts centralisateurs de lait de femme (F.M.S.) ont leur raison d'être dans la lutte contre la mortalité infantile.

La fondatrice des F.M.S., M. E. KAYSER, a pourtant toujours bien répété que le lait de femme stérilisé des dépôts centralisateurs se range derrière le lait tété, et qu'il est malgré les affirmations de ses adversaires meilleur que le lait de vache bouilli et ses mélanges. Il a été prouvé par nos essais et ceux d'autres expérimentateurs sur des jumeaux univitellins que l'affirmation des adversaires du lait de femme que celui-ci est nuisible aux enfants n'est pas justifiée.

Pour que le lait des F.M.S. ne nuise pas aux enfants, M. E. KAYSER estime que trois conditions doivent être remplies dans la livraison du lait de femme aux F.M.S.

a) Le lait de femme ne doit subir aucune sorte d'addition non voulue (en premier lieu, pas de lait étranger, d'eau et analogues);

b) Le lait ne doit avoir subi aucune espèce de décomposition (détermination de l'acidité et du $p \mathrm{H}$ ) ;

c) Le lait doit être traité au F.M.S. de telle façon qu'aucune modification de ce lait ne puisse nuire aux enfants.

Pour remplir ces trois eonditions, M. E. KAYSER a perfectionné les méthodes les plus diverses de recherches et de conservation du

(1) Zentralblatt für Gynäkologie, 1952, fascicule 12. 